\title{
IVERSEN'S FORMULA FOR THE SECOND CHERN CLASSES OF REGULAR SURFACES IN ANY CHARACTERISTIC
}

\author{
I. B. ZHUKOV \\ Dedicated to the 100th anniversary of D. K. Faddeev's birth \\ Abstract. The formula mentioned in the title is proved.
}

\section{INTRODUCTION}

Let $S, T$ be complete nonsingular surfaces over an algebraically closed field $k$ of any characteristic, and let $h: T \rightarrow S$ be a finite separable morphism of degree $n$. We establish a formula that expresses the Euler characteristic (understood as the degree of the second Chern class $\int c_{2, T}$ ) of $T$ via the Euler characteristic of $S$ and some local terms associated with components of the branch divisor $B_{h}=h^{*} R_{h}$ of $h$ and with certain points on $B_{h}$ (here $R_{h}$ is the ramification divisor).

Let $B_{h}=\sum_{i} b_{i} B_{i}$, where the $B_{i}$ are prime divisors on $S$. Then

$$
\chi_{T}-n \chi_{S}=\sum_{i} b_{i} \chi_{B_{i}}+\sum_{Q} \lambda_{f}\left(\widehat{\mathcal{O}_{T, Q}} / \widehat{\mathcal{O}_{S, h(Q)}}\right) \text {. }
$$

Here $Q$ runs over the closed points of $T$, and $\lambda_{f}\left(A^{\prime} / A\right)$ is a certain invariant defined explicitly for an extension of complete 2-dimensional regular local rings $A^{\prime} / A$ and for a (sufficiently general) element $f$ of the maximal ideal of $A$; this element must be a local equation of a curve at $h(Q)$ in any fixed sufficiently good pencil of curves on $S$. This invariant is defined in terms of the different of all $A^{\prime} / \mathfrak{q}$ over $A /(\mathfrak{q} \cap A)$, the invariants of singularity of arcs corresponding to $A^{\prime} / \mathfrak{q}$ and $A /(\mathfrak{q} \cap A)$, and the invariants of intersection of the latter arc with the branch divisor, where $\mathfrak{q}$ runs over the prime divisors of $f$ in $A^{\prime}$. (For the precise statement, see the definitions in $\S \$ 1,3$, 4 and Theorem 7.4.)

The term $\lambda_{f}\left(\widehat{\mathcal{O}_{T, Q}} / \widehat{\mathcal{O}_{S, h(Q)}}\right)$ does not vanish only for a finite number of points $Q$, all of them lying on the ramification divisor of $f$.

What is also important, this term depends on the infinitesimal (rather than merely local) behavior of $h$, i.e., on the properties of extensions of completed local rings, and this reduces the further analysis to some questions related only to complete regular local rings.

The formula under discussion is a 2-dimensional analog of the Riemann-Hurwitz formula. In characteristic 0 it was established by Iversen in [V].

Remark 0.0.1. I was not able to avoid the dependence on $f$ in the definition of the term $\lambda$ that describes ramification in codimension 2. However, I expect that $\lambda_{f}$ is independent of $f$ (and, therefore, the formula is in its final form) in case there is no ferocious ramification. (This condition means that all morphisms of curves induced by the given finite morphism of surfaces are separable.)

2000 Mathematics Subject Classification. Primary 14J29.

Key words and phrases. Euler characteristic, Chern class, ferocious ramification, wild different. 
In the good (nonferocious) case, we can show directly that, at all "nonexceptional" points, $\lambda_{f}$ does not depend on the choice of a pencil of curves if the pencils are "sufficiently general". Then the exceptional points can be managed presumably by a local-global argument such as that in $[\mathrm{L}]$.

A considerable part of this research was done during the author's stay at Humboldt Univerität zu Berlin under the support of the Alexander von Humboldt Foundation and at the Max-Planck-Institut für Mathematik in Bonn. The work was completed under the support of the Rückkehrstipendium of the Humboldt Foundation and INTAS grant. I am very much grateful to all these organizations. Also, I would like to thank A. N. Parshin and H. Kurke for numerous inspiring discussions.

\section{§1. Definitions, notation, AND PRELiminary FACTS}

For an arbitrary domain $A$, we denote by $\tilde{A}$ the integral closure of $A ; \delta(A)=l_{A}(\tilde{A} / A)$; $\nu(A)$ is the number of maximal prime ideals in $\tilde{A} ; \mathfrak{q}_{A}$ is the conductor of $A$, i.e., $\{c \in \tilde{A} \mid$ $c \tilde{A} \subset A\} ; Q(A)$ is the field of fractions.

If $C$ is a reduced irreducible curve and $P$ is a closed point on it, we denote $\nu_{P}(C)=$ $\nu\left(\mathcal{O}_{C, P}\right)$.

If $A$ is a 1-dimensional domain, $a \in A$, and $a \neq 0$, we denote $\operatorname{ord}_{A} a=l_{A}(A / a A)$.

If $A$ is a 1-dimensional local domain, $\omega \in \Omega_{A}$, and $v$ is the valuation in $\tilde{A}$, we denote $v(\omega)=v(g)$, where $\omega=g d t$, and $t$ is any prime element of $\tilde{A}$.

If $A$ is a local ring, we denote by $\mathfrak{m}_{A}$ the maximal ideal of $A$ and by $\widehat{A}$ the completion of $A$.

$S_{i}$ denotes the set of $i$-dimensional points of a scheme $S$.

$k(S)$ denotes the field of rational functions on an integral scheme $S$.

Let $C$ be a divisor on a complete regular surface $S$ over a perfect field $k$. Its arithmetic genus is defined as

$$
p_{a}(C)=1+\frac{1}{2}\left(C+K_{S} \cdot C\right) .
$$

Lemma 1.1. Let $A$ be a 1-dimensional local domain such that $\tilde{A}$ is finite over $A$. Let $\mathfrak{m}_{1}, \ldots, \mathfrak{m}_{n}$ be all maximal ideals of $\tilde{A}$. Then for any $a \in A, a \neq 0$, we have

$$
\operatorname{ord}_{A} a=\sum_{i=1}^{n} \operatorname{ord}_{\tilde{A}_{\mathfrak{m}_{i}}} a \cdot\left[\tilde{A}_{\mathfrak{m}_{i}} / \mathfrak{m}_{i} \tilde{A}_{\mathfrak{m}_{i}}: A / \mathfrak{m}_{A}\right] .
$$

Proof. See [F] Example A.3.1].

Corollary 1.1.1. Under the assumptions of Lemma 1.1, suppose that $A$ is a $k$-algebra, where $k$ is a field. Then

$$
\operatorname{dim}_{k}(A / a)=\sum_{i=1}^{n} \operatorname{dim}_{k}\left(\widetilde{A_{\mathfrak{m}_{i}}} / a\right) .
$$

Wild different. Let $B / A$ be a finite separable extension of complete discrete valuation rings. The order of the different $\mathfrak{D}_{B / A}$ can be written in the form

$$
v_{B}\left(\mathfrak{D}_{B / A}\right)=e_{B / A}-1+d(B \mid A),
$$

where $d(B \mid A) \geq 0$, and $d(B \mid A)=0$ if and only if the extension is tame. The term $d(B \mid A)$ is called the wild different of $B / A$; we agree that $d(B \mid A)=\infty$ if $B / A$ is nonseparable.

Let $\mathcal{O}$ be a complete 2-dimensional regular local ring with a coefficient subfield $k$. Suppose $b \in \mathcal{O}$ and $a \in \mathfrak{m} \backslash\{0\}$, where $\mathfrak{m}$ is the maximal ideal of $\mathcal{O}$. In this situation we introduce the quantity $d(a, b) \in \mathbb{N} \cup\{+\infty\}$. 
If $b$ is an irreducible element, we denote by $\bar{a}$ the image of $a$ in $B=\widetilde{\mathcal{O} / b}$. Then

$$
d(a, b)=d(B \mid k[[\bar{a}]])
$$

if $\bar{a} \neq 0$, and $d(a, b)=+\infty$ otherwise. In the general case, if $b=\varepsilon \prod_{i} p_{i}^{r_{i}}$ is a canonical factorization, we put

$$
d(a, b)=\sum_{i} r_{i} d\left(a, p_{i}\right) .
$$

Let $A$ be a complete discrete valuation ring with a coefficient subfield $k$ and valuation $v$.

Lemma 1.2. For any $f \in \mathfrak{m}_{A}, f \neq 0$, we have $v(d f)=v(f)-1+d(A \mid k[[f]])$.

Proof. Choose an arbitrary prime element $\pi \in A$. Then $A=A_{0}[\pi]$, where $A_{0}=k[[f]]$; $f=F(\pi)$ for some $F \in X k[[X]]$. Let $F_{0} \in A_{0}[X]$ be the characteristic polynomial of $\pi$. Then $f-F(\pi)=0$ implies $F(X)-f=F_{0}(X) \rho(X)$ for some $\rho \in A_{0}[[X]]$, and $v_{A_{0}}(f)=v_{A_{0}}\left(F_{0}(0)\right)=1$ implies $v_{A_{0}}(\rho(0))=0$. We obtain

$$
F^{\prime}(X)=F_{0}^{\prime}(X) \rho+F_{0} \rho^{\prime}
$$

and $F^{\prime}(\pi)=F_{0}^{\prime}(\pi) \rho(\pi)$. Therefore,

$$
v(d f)=v\left(F^{\prime}(\pi) d \pi\right)=v\left(F^{\prime}(\pi)\right)=v\left(F_{0}^{\prime}(\pi)\right)=v\left(\mathfrak{D}_{A / A_{0}}\right)
$$

by [Se, Chapter III, Proposition 11, Corollary 2].

Finite determinacy. Let $k$ be an algebraically closed field, let $\mathfrak{m}$ be the maximal ideal of $k\left[\left[X_{0}, \ldots, X_{n}\right]\right]$, and let $f \in \mathfrak{m}$. The Tyurina ideal of $f$ is defined as follows:

$$
j(f)=\left(f, \frac{\partial f}{\partial X_{0}}, \ldots, \frac{\partial f}{\partial X_{n}}\right) .
$$

Proposition 1.3. Suppose $f \in \mathfrak{m}$ and $\mathfrak{m}^{l} \subset j(f)$. Then for any $g \equiv f \bmod \mathfrak{m}^{2 l+1}$ we have $k[[X, Y]] /(f) \cong k[[X, Y]] /(g)$.

Proof. This is exactly [GK Lemma 2.6].

Generalization of the Weierstrass preparation lemma. Here $k$ is an arbitrary perfect field.

Lemma 1.4. Let $f \in k[[X, Y]]$ be irreducible. Then, after a possible exchange of $X$ and $Y$, we have $f=u f_{0}$, where $u \in k[[X, Y]]^{*}$, and

$$
f_{0}=Y^{n}+a_{1} Y^{n-1}+\cdots+a_{n-1} Y+a_{n}
$$

is a separable polynomial in $Y$, where $a_{i} \in X k[[X]]$.

Proof. This is exactly [Iv, Lemma 2.4].

Corollary 1.4.1. Let $\mathcal{O}$ be a complete 2-dimensional local ring with coefficient subfield $k$, and let $\pi$ be an irreducible element of $\mathcal{O}$. Then $d \pi \notin \pi \Omega_{\mathcal{O} / k}$.

Proof. Since $\Omega_{\mathcal{O} / k}$ is a free $\mathcal{O}$-module with a basis $d s, d t$, an equivalent statement is that either $\pi \nmid \frac{\partial \pi}{\partial s}$, or $\pi \nmid \frac{\partial \pi}{\partial t}$. But this follows from Lemma 1.4 .

Ramification divisor and branch divisor. For a finite separable morphism of twodimensional schemes $h: T \rightarrow S$ of degree $n$, we consider the corresponding ramification divisor

$$
R_{h}=\sum_{\eta \in T_{1}} l_{\mathcal{O}_{T, \eta}}\left(\Omega_{T / S, \eta}\right) \cdot D_{\eta},
$$

where $D_{\eta}$ is a prime divisor with the generic point $\eta$, and the branch divisor

$$
B_{h}=f_{*} R_{h} .
$$




\section{§2. AnAlytic ADJUnCTION FORMUla}

Let $A$ be a 1-dimensional complete local domain with an algebraically closed coefficient subfield $k$. Assume that emb $\operatorname{dim} A \leq 2$. In other words, $A$ is isomorphic to $k[[X, Y]] /(f)$, where $f$ is an irreducible element of the maximal ideal of $k[[X, Y]]$.

Proposition 2.1. There exists an irreducible polynomial $f \in X k[X, Y]+Y k[X, Y]$ such that $A$ is isomorphic to $k[[X, Y]] /(f)$.

Proof. By Corollary [1.4.1, the Tyurina ideal $j(f)$ is an ideal of definition in $k[[X, Y]]$, and we are done by Proposition 1.3 .

Proposition 2.2. We have

$$
\operatorname{dim}_{k}\left(\tilde{A} / \mathfrak{q}_{A}\right)=2 \delta(A) .
$$

Proof. Let $A_{0}=(k[X, Y] /(f))_{(X, Y)}$, where $f$ is as in Proposition 2.1. Then $A$ is the completion of $A_{0}, \operatorname{dim}_{k}\left(\tilde{A} / \mathfrak{q}_{A}\right)=\operatorname{dim}_{k}\left(\widetilde{A_{0}} / \mathfrak{q}_{A_{0}}\right)$, and $\delta(A)=\delta\left(A_{0}\right)$. It remains to show that $\operatorname{dim}_{k}\left(\widetilde{A_{0}} / \mathfrak{q}_{A_{0}}\right)=2 \delta\left(A_{0}\right)$, but this is [Sa, Theorem 5].

Let $s, t$ be generators of the maximal ideal of $A$ such that $f \nmid \frac{\partial f}{\partial t}$, let $v$ be the valuation in $\tilde{A}$, and let $\bar{x}$ be the class of $x \in k[[X, Y]]$ in $A$.

Proposition 2.3. We have $v(d \bar{s})<\infty$, and

$$
v\left(\mathfrak{q}_{A}\right)+v(d \bar{s})=v\left(\frac{\partial f}{\partial t}\right) .
$$

Proof. Indeed, $\operatorname{dim}_{k}\left(\tilde{A} / \mathfrak{q}_{A}\right)=\operatorname{dim}_{k}\left(\widetilde{A_{0}} / \mathfrak{q}_{A_{0}}\right)$ as in the preceding proof; we may assume that $s, t \in A_{0}$. It remains to apply [Sa, Theorem $\left.3 \mathrm{bis}\right]$.

Theorem 2.4. Let $s, t$ be as above. Then

$$
2 \delta(A)+v(d \bar{s})=v\left(\frac{\overline{\partial f}}{\partial t}\right) .
$$

Proof. This follows from Propositions 2.2 and 2.3 .

\section{$\S 3$. TAME AND WILD SINGULARITY}

In this section $k$ is an algebraically closed field, $\mathcal{O}$ is a 2-dimensional complete regular local ring with the coefficient field $k$, and $K$ is the fraction field of $\mathcal{O}$. If $f, g \in \mathcal{O}$ are such that $(f, g)$ is an ideal of definition, we denote $(f . g)=\operatorname{dim}_{k} \mathcal{O} /(f, g)$; it is easy to verify the bimultiplicativity of $(f . g)$.

Let $\pi_{1}, \ldots, \pi_{r}$ be pairwise nonassociated prime elements of $\mathcal{O}$, and let $f=\pi_{1} \ldots \pi_{r}$. We introduce the tame singularity of $f$ :

$$
\operatorname{sing}_{\mathcal{O}}^{t} f=2 \sum_{i} \delta\left(\mathcal{O} / \pi_{i}\right)-r+\sum_{i \neq j}\left(\pi_{i} . \pi_{j}\right)+1 .
$$

Next, we introduce the wild singularity $\operatorname{sing}_{\mathcal{O}}^{w} f$ of $f$. Assume that there exist regular local parameters $s, t$ of $\mathcal{O}$ such that

(i) $d(f, s), d(s, f), d\left(f, \frac{\partial f}{\partial t}\right), d\left(s, \frac{\partial f}{\partial t}\right)$ are all finite, and

(ii) $\pi_{i} \nmid \frac{\partial \pi_{i}}{\partial t}$ for any $i ; s \nmid \frac{\partial f}{\partial t}$.

Then

$$
\operatorname{sing}_{\mathcal{O}}^{w} f=-d(f, s)+d(s, f)+d\left(f, \frac{\partial f}{\partial t}\right)-d\left(s, \frac{\partial f}{\partial t}\right) .
$$


(In Corollary 3.3.1 we shall see that this value is independent of the choice of $s, t$.) If no required $s, t$ exist, we put $\operatorname{sing}_{\mathcal{O}}^{w} f=\infty$.

Our plan is to express the Milnor number $\mu(f)=\left(\frac{\partial f}{\partial s} \cdot \frac{\partial f}{\partial t}\right)$ as the sum of tame and wild singularities. It is easily seen that this value is independent of the choice of $s$ and $t$.

Remark 3.0.1. In the prime characteristic case, the Milnor number is not an invariant of $\mathcal{O} /(f)$, i.e., $\varepsilon \in \mathcal{O}^{*}$ does not imply $\mu(\varepsilon f)=\mu(f)$ (see [GK, Subsection 2.1]). However, it is easy to show that $\varepsilon \in k[[f]]^{*}$ implies $\mu(\varepsilon f)=\mu(f)$.

Lemma 3.1. The local parameters $s, t$ in $\mathcal{O}$ can be chosen so that

1) $\pi_{i} \nmid \frac{\partial \pi_{i}}{\partial t}$ for every $i$;

2) $s \nmid \frac{\partial f}{\partial t}$;

3) if char $k=p>0$, then the conditions $s^{\prime} \equiv s \bmod (s, t)^{2}$ and $s^{\prime} \in k\left[\left[s, t^{p}\right]\right]$ imply $s^{\prime} \nmid \frac{\partial f}{\partial t}$.

Proof. Let $s_{0}$ and $t_{0}$ be arbitrary regular local parameters in $\mathcal{O}$. Put

$$
\begin{aligned}
& s=s_{0}+\alpha t_{0}+\beta t_{0}^{2}, \\
& t=t_{0},
\end{aligned}
$$

where $\alpha, \beta$ are some elements of $k$. It is easily seen that

$$
\begin{aligned}
& \frac{\partial}{\partial s}=\frac{\partial}{\partial s_{0}}, \\
& \frac{\partial}{\partial t}=\frac{\partial}{\partial t_{0}}-\alpha \frac{\partial}{\partial s_{0}}-2 \beta t \frac{\partial}{\partial s_{0}} .
\end{aligned}
$$

By Corollary 1.4.1, we have $\pi_{i} \nmid d \pi_{i}$ for any $i$, i.e., $\pi_{i} \nmid \frac{\partial \pi_{i}}{\partial s_{0}}$ or $\pi_{i} \nmid \frac{\partial \pi_{i}}{\partial t_{0}}$. It follows that the set $M$ of all pairs $(\alpha, \beta)$ satisfying condition 1$)$ is nonempty (because $k$ is infinite) and open on the plane.

After a possible intermediate change of variables, we may assume that $(0,0) \in M$. The relation $\pi_{i} \nmid \frac{\partial \pi_{i}}{\partial t_{0}}$ implies that $\pi_{i} \nmid \frac{\partial f}{\partial t_{0}}$, whence $d f \neq 0$. Let $f_{N}$ denote the form in $f$ of minimal degree such that $d f_{N} \neq 0$. Observe that either of the conditions $s \mid \frac{\partial f}{\partial t}$ and $s^{\prime} \mid \frac{\partial f}{\partial t}$ implies

$$
s \mid \frac{\partial f_{N}}{\partial t}+\frac{\partial f_{N+1}}{\partial t}
$$

in $\mathcal{O} /\left(s_{0}, t_{0}\right)^{N+1}$; in this ring we put

$$
\frac{\partial f_{N}}{\partial t}+\frac{\partial f_{N+1}}{\partial t}=s \cdot\left(\sum_{i=0}^{N-2} g_{i} s_{0}^{N-2-i} t_{0}^{i}+\sum_{i=0}^{N-1} h_{i} s_{0}^{N-1-i} t_{0}^{i}\right)
$$

After the substitution $s=s_{0}+\alpha t_{0}+\beta t_{0}^{2}$, we obtain

$$
\frac{\partial f}{\partial t}=\sum_{i=0}^{N-1}\left(g_{i}+\alpha g_{i-1}\right) s_{0}^{N-1-i} t_{0}^{i}+\sum_{i=0}^{N}\left(h_{i}+\alpha h_{i-1}+\beta g_{i-2}\right) s_{0}^{N-i} t_{0}^{i} .
$$


On the other hand, put

$$
\begin{aligned}
f_{N} & =\sum_{i=0}^{N} a_{i} s_{0}^{N-i} t_{0}^{i} \\
f_{N+1} & =\sum_{i=0}^{N+1} b_{i} s_{0}^{N+1-i} t_{0}^{i} .
\end{aligned}
$$

In the same factor ring we compute

$$
\begin{aligned}
\frac{\partial f}{\partial t}= & \sum_{i=0}^{N-1}\left((i+1) a_{i+1}-\alpha(N-i) a_{i}\right) s_{0}^{N-1-i} t_{0}^{i} \\
& -\sum_{i=0}^{N}\left((N-i-1) b_{i+1}+\alpha(N-i+1) b_{i}+2 \beta(N-i+1) a_{i-1}\right) s_{0}^{N-i} t_{0}^{i} .
\end{aligned}
$$

Note that the $g_{i}$ and $h_{i}$ (but not $a_{i}$ and $b_{i}$ ) depend on $\alpha$ and $\beta$.

Comparing forms of degree $N-1$, we obtain the following system of relations:

$$
\begin{aligned}
g_{0} & =a_{1}-\alpha \cdot N a_{0}, \\
g_{1}+\alpha g_{0} & =2 a_{2}-\alpha \cdot(N-1) a_{1}, \\
g_{2}+\alpha g_{1} & =3 a_{3}-\alpha \cdot(N-2) a_{2}, \\
& \ldots \\
g_{N-2}+\alpha g_{N-3} & =(N-1) a_{N-1}-\alpha \cdot 2 a_{N-2}, \\
\alpha g_{N-2} & =N a_{N}-\alpha \cdot a_{N-1},
\end{aligned}
$$

whence

$$
N\left(a_{n}-\alpha a_{N-1}+\alpha^{2} a_{N-2}-\cdots \pm \alpha^{N} a_{0}\right)=0 .
$$

Suppose that the statement to be proved is false; this means in particular that (1) is satisfied for all $\alpha$ such that $(\alpha, 0) \in M$, i.e., for infinitely many values of $\alpha$. Then (2) is valid for infinitely many $\alpha$ 's, and we see that $N=0$ in $k$. This concludes the proof in the case where char $k=0$, whereas for char $k=p>0$ we obtain $p \mid N$, whence

$$
g_{i}=(i+1) a_{i+1}, \quad i=0,1, \ldots, N-2 .
$$

Next, comparing forms of degree $N$, we obtain the system of relations

$$
h_{i}+\alpha h_{i-1}+\beta g_{i-2}=(N-i-1) b_{i+1}+\alpha(N-i+1) b_{i}+2 \beta(N-i+1) a_{i-1},
$$

$i=0, \ldots, N$ (here we agree that $g_{-2}=g_{-1}=0$, etc.). Assuming $\beta=0$, we obtain

$$
b_{N+1}-\alpha b_{N}+\alpha^{2} b_{N-1}-\cdots \pm \alpha^{N+1} b_{0}=0
$$

for infinitely many values of $\alpha$, whence $b_{i}=0$ for any $i$.

Finally, let $\beta$ take any nonzero value such that $(\alpha, \beta) \in M$ for infinitely many values of $\alpha$. Relations (3) and (4) imply that

$$
h_{i}+\alpha h_{i-1}=(-3) \beta(i-1) a_{i-1},
$$

$i=0, \ldots, N$. Therefore

$$
\sum_{i=0}^{N-1}(-1)^{i}(N-1-i) \beta a_{N-1-i} \alpha^{i}=0 .
$$

Since the value of a polynomial vanishes for infinitely many values of $\alpha$, all the coefficients must be zero, i.e., $a_{n}=0$ if $p \nmid n$. We obtain $f_{N} \in k\left[\left[s_{0}^{p}, t_{0}^{p}\right]\right]$, in contradiction with $d f_{N} \neq 0$. 
Lemma 3.2. Suppose $s, t$ are regular local parameters, $q$ is an irreducible element of $\mathcal{O}$, and $q \mid \frac{\partial q}{\partial t}$. Then $q=q_{1} \varepsilon$, where $q_{1} \in k[[s]][t], \varepsilon \in \mathcal{O}^{*}$, and $\frac{\partial q_{1}}{\partial t}=0$.

Proof. This follows immediately from the Weierstrass preparation lemma.

Corollary 3.2.1. Let $s, t$ be regular local parameters, and let $\mathfrak{q}$ be a prime ideal of height 1 in $\mathcal{O}$ such that $d \bar{s}=0$ in $\Omega_{\mathcal{O} / \mathfrak{q}}$, where $\bar{s}$ is the class of $s$ in $\mathcal{O} / \mathfrak{q}$. Then the ideal $\mathfrak{q}$ has a generator $q$ such that $\frac{\partial q}{\partial t}=0$ and $q \equiv s \bmod (s, t)^{2}$.

Proof. Let $q_{0}$ be an arbitrary generator of $\mathfrak{q}$; then $d q_{0}=\frac{\partial q_{0}}{\partial s} d s+\frac{\partial q_{0}}{\partial t} d t$ implies $\overline{\left(\frac{\partial q_{0}}{\partial t}\right)} \cdot d \bar{t}=$ 0 , whence $\overline{\left(\frac{\partial q_{0}}{\partial t}\right)}=0$.

Proposition 3.3. Let $s, t$ be any regular local parameters of $\mathcal{O}$ satisfying the conditions of Lemma 3.1, Assume that $\mu(f)<\infty$. Then $d(f, s), d(s, f), d\left(f, \frac{\partial f}{\partial t}\right)$, and $d\left(s, \frac{\partial f}{\partial t}\right)$ are all finite, and

$$
\mu(f)=\operatorname{sing}_{\mathcal{O}}^{t} f-d(f, s)+d(s, f)+d\left(f, \frac{\partial f}{\partial t}\right)-d\left(s, \frac{\partial f}{\partial t}\right) .
$$

Proof. First, we show that $d\left(f, \frac{\partial f}{\partial t}\right)<\infty, d\left(s, \frac{\partial f}{\partial t}\right)<\infty$, and

$$
\mu(f)=\left(f \cdot \frac{\partial f}{\partial t}\right)+d\left(f, \frac{\partial f}{\partial t}\right)-\left(s \cdot \frac{\partial f}{\partial t}\right)-d\left(s, \frac{\partial f}{\partial t}\right) .
$$

It suffices to check that $d(f, q)<\infty, d(s, q)<\infty$, and

$$
(f \cdot q)=\left(\frac{\partial f}{\partial s} \cdot q\right)-d(f, q)+(s \cdot q)+d(s, q)
$$

where $q$ is any irreducible divisor of $\frac{\partial f}{\partial t}$. It is a commonplace that all other terms on both sides of the relation are also finite.

By Corollary 1.1.1, for any $a \in \mathcal{O}$ such that $q \nmid a$ we have $(a . q)=v(\bar{a})$, where $\bar{a}$ is the class of $a$ in $\mathcal{O} / q$, and $v$ is the valuation in $\widetilde{\mathcal{O} / q}$. Since $d f=\frac{\partial f}{\partial s} d s+\frac{\partial f}{\partial t} d t$, we obtain $d \bar{f}=\overline{\frac{\partial f}{\partial s}} d \bar{s}$ in $\Omega_{\mathcal{O} / q}$.

Suppose that $d \bar{s}=0$ in $\mathcal{O} / q$. Only the case where char $k=p>0$ is nontrivial. By Corollary 3.2.1, a generator $s^{\prime}$ of the ideal $(q)$ can be chosen so that $s^{\prime} \equiv s \bmod (s, t)^{2}$, $s^{\prime} \in k\left[\left[s, t^{p}\right]\right]$. Then by Lemma 3.1 we obtain $s^{\prime} \nmid \frac{\partial f}{\partial t}$, in contradiction with $q \mid \frac{\partial f}{\partial t}$.

Thus, $v(d \bar{s})<\infty$ and $v(d \bar{f})<\infty$. Applying Lemma 1.2 twice, we see that $d(f, q)$ and $d(s, q)$ are finite, and

$$
\begin{aligned}
v(\bar{f}) & =v(d \bar{f})+1-d(f, q) \\
& =v\left(\frac{\overline{\partial f}}{\partial s}\right)+v(d \bar{s})+1-d(f, q) \\
& =v\left(\frac{\partial f}{\partial s}\right)+v(\bar{s})-d(f, q)+d(s, q),
\end{aligned}
$$

which proves (6) and (5).

Similarly, considering $\Omega_{\mathcal{O} / s}$ instead of $\Omega_{\mathcal{O} / q}$, we obtain $d(f, s)<\infty$ and

$$
(f . s)-1=\left(\frac{\partial f}{\partial t} \cdot s\right)-d(f, s) .
$$


We combine (5) and (7):

$$
\mu(f)=\left(f \cdot \frac{\partial f}{\partial t}\right)-((f . s)-1)+d\left(f, \frac{\partial f}{\partial t}\right)-d\left(s, \frac{\partial f}{\partial t}\right)-d(f, s) .
$$

It remains to show that $d(s, f)<\infty$, and

$$
\left(f \cdot \frac{\partial f}{\partial t}\right)-((f . s)-1)=d(s, f)+\operatorname{sing}_{\mathcal{O}}^{t} f .
$$

It suffices to check the relation

$$
\left(\pi_{i} \cdot \frac{\partial \pi_{i}}{\partial t}\right)-\left(\pi_{i} . s\right)=2 \delta\left(\mathcal{O} / \pi_{i}\right)-1+d\left(s, \pi_{i}\right)
$$

for every $i$. By Corollary 1.1.1 this is equivalent to

$$
v\left(\overline{\frac{\partial \pi_{i}}{\partial t}}\right)-v(\bar{s})+1-d\left(s, \pi_{i}\right)=2 \delta\left(\mathcal{O} / \pi_{i}\right)
$$

where $v$ is the valuation in $\widetilde{\mathcal{O} / \pi_{i}}$. By Lemma 1.2, $d \bar{s} \neq 0$ implies $d\left(s, \pi_{i}\right)<\infty$, and (8) can be rewritten as

$$
v\left(\overline{\frac{\partial \pi_{i}}{\partial t}}\right)-v(d \bar{s})=2 \delta\left(\mathcal{O} / \pi_{i}\right) .
$$

However, this identity is none other than the analytic adjunction formula of Theorem 2.4 for the ring $\mathcal{O} / \pi_{i}$.

Corollary 3.3.1. $\operatorname{sing}_{\mathcal{O}}^{w} f$ is independent of the choice of $s, t$.

Examples. Let $x, y$ be any system of regular local parameters in $\mathcal{O}$.

1. Let $f=x^{l}-y^{m}, p \nmid l, p \nmid m$. Then $\operatorname{sing}_{\mathcal{O}}^{w} f=0$.

2. Let $f=y^{p}+y^{M}-x^{2}, p \nmid M$. Taking $s=x, t=y$, we compute

$$
d(f, s)=d(s, f)=d\left(s, \frac{\partial f}{\partial t}\right)=0,
$$

and

$$
d\left(f, \frac{\partial f}{\partial t}\right)=M-p
$$

whence $\operatorname{sing}_{\mathcal{O}}^{w} f=M-p$.

3. Let $f=y^{p}-x^{2}$. Then $d(f, x)=\infty$, whence for any choice of regular local parameters $s, t$ we have $d\left(f, \frac{\partial f}{\partial t}\right)=\infty$, and $\operatorname{sing}_{\mathcal{O}}^{w} f=\infty$.

\section{§4. Extensions of 2-Dimensional COMPLETE REgUlAR LOCAL RINGS}

In this section $\mathcal{O}^{\prime} / \mathcal{O}$ is a finite separable extension of complete 2-dimensional regular local rings of some degree $n$, both having an algebraically closed coefficient subfield $k$.

Proposition 4.1. Let $\pi$ be a prime element of $\mathcal{O}$ that does not divide a local equation $\beta$ of the branch divisor of $\mathcal{O}^{\prime} / \mathcal{O}$, and let $\pi=\pi_{1} \ldots \pi_{r}$, where $\pi_{1}, \ldots, \pi_{r}$ are irreducible elements of $\mathcal{O}^{\prime}$. Then

$$
\operatorname{sing}_{\mathcal{O}^{\prime}}^{t} \pi-n \operatorname{sing}_{\mathcal{O}}^{t} \pi=(\beta . \pi)-(n-1)-\sum_{i=1}^{r} d\left(\left(\mathcal{O}^{\prime} / \pi_{i}\right) \mid(\mathcal{O} / \pi)\right) .
$$


Proof. We choose regular local parameters $s, t$ in $\mathcal{O}$ so that $\pi \nmid \frac{\partial \pi}{\partial t}$. This can be done by Lemma 3.1 Fixing $j$, we choose regular local parameters $u, v$ in $\mathcal{O}^{\prime}$ so that $\pi_{j} \nmid \frac{\partial \pi_{j}}{\partial v}$. Denote $A_{j}=\widetilde{\mathcal{O}^{\prime} / \pi_{j}}$.

Now we do some computations in $\Omega_{Q\left(A_{j}\right) / k}$; the elements of $\mathcal{O}^{\prime}$ (respectively, of $\Omega_{\mathcal{O}^{\prime} / k}$ ) will be denoted by the same letters as their images in $A_{j}$ (respectively, in $\Omega_{Q\left(A_{j}\right) / k}$ ). First, we have

$$
\frac{\partial \pi_{j}}{\partial u} d u+\frac{\partial \pi_{j}}{\partial v} d v=d \pi_{j}=0
$$

Next, the derivations $\frac{\partial}{\partial s}$ and $\frac{\partial}{\partial t}$ of $Q(\mathcal{O})$ can be uniquely extended up to continuous derivations of $Q\left(\mathcal{O}^{\prime}\right)$, also denoted by $\frac{\partial}{\partial s}$ and $\frac{\partial}{\partial t}$. We have

$$
\frac{\partial}{\partial u}=\frac{\partial s}{\partial u} \cdot \frac{\partial}{\partial s}+\frac{\partial t}{\partial u} \cdot \frac{\partial}{\partial t}
$$

because these derivations coincide on the elements $s$ and $t$, and $Q\left(\mathcal{O}^{\prime}\right)$ is separable over $Q(\mathcal{O})=Q(k[[s, t]])$. Therefore, the identity

$$
\begin{aligned}
d s & =\left(\frac{\partial s}{\partial u} \frac{\partial \pi_{j}}{\partial v} d u+\frac{\partial s}{\partial v} \frac{\partial \pi_{j}}{\partial v} d v\right)\left(\frac{\partial \pi_{j}}{\partial v}\right)^{-1} \\
& =\left(\frac{\partial s}{\partial u} \frac{\partial \pi_{j}}{\partial v} d u-\frac{\partial s}{\partial v} \frac{\partial \pi_{j}}{\partial u} d u\right)\left(\frac{\partial \pi_{j}}{\partial v}\right)^{-1} \\
& =\left|\begin{array}{ll}
\frac{\partial s}{\partial u} & \frac{\partial s}{\partial v} \\
\frac{\partial \pi_{j}}{\partial u} & \frac{\partial \pi_{j}}{\partial v}
\end{array}\right|\left(\frac{\partial \pi_{j}}{\partial v}\right)^{-1} d u
\end{aligned}
$$

in $\Omega_{Q\left(A_{j}\right) / k}$ implies

$$
\begin{aligned}
\left(\frac{\partial \pi}{\partial t}\right)^{-1} d s & =\left(\frac{\partial \pi}{\partial t}\right)^{-1}\left|\begin{array}{cc}
\frac{\partial s}{\partial s} & \frac{\partial s}{\partial t} \\
\frac{\partial \pi_{j}}{\partial s} & \frac{\partial \pi_{j}}{\partial t}
\end{array}\right|\left|\begin{array}{cc}
\frac{\partial s}{\partial u} & \frac{\partial s}{\partial v} \\
\frac{\partial t}{\partial u} & \frac{\partial t}{\partial v}
\end{array}\right|\left(\frac{\partial \pi_{j}}{\partial v}\right)^{-1} d u \\
& =\left(\frac{\partial \pi}{\partial t}\right)^{-1} \frac{\partial \pi_{j}}{\partial t}\left|\begin{array}{cc}
\frac{\partial s}{\partial u} & \frac{\partial s}{\partial v} \\
\frac{\partial t}{\partial u} & \frac{\partial t}{\partial v}
\end{array}\right|\left(\frac{\partial \pi_{j}}{\partial v}\right)^{-1} d u \\
& =\prod_{i \neq j} \pi_{i}^{-1}\left|\begin{array}{cc}
\frac{\partial s}{\partial u} & \frac{\partial s}{\partial v} \\
\frac{\partial t}{\partial u} & \frac{\partial t}{\partial v}
\end{array}\right|\left(\frac{\partial \pi_{j}}{\partial v}\right)^{-1} d u .
\end{aligned}
$$

We shall apply the analytic adjunction formula of Theorem 2.4. Let $w_{j}$ and $w$ be the valuations in $A_{j}$ and $A=\widetilde{\mathcal{O} / \pi}$, respectively, and let $e_{j}=e\left(A_{j} / A\right), d_{j}=d\left(A_{j} \mid A\right)$. We denote by $\rho_{j}$ and $\rho$ any local parameters in $A_{j}$ and $A$, respectively. We write the lefthand side and the right-hand side of (9) in the form $L d \rho_{j}=L_{0} d \rho$ and $R d \rho_{j}$, respectively, and compute the valuations of $L$ and $R$.

From the exact sequence

$$
\Omega_{A / k} \otimes_{A} A_{j} \rightarrow \Omega_{A_{j} / k} \rightarrow \Omega_{A_{j} / A} \rightarrow 0
$$

it is clear that $d \rho=a d \rho_{j}$, where $a$ is a generator of the different of $A_{j} / A$, that is, $w_{j}(a)=e_{j}-1+d_{j}$. We have $w\left(L_{0}\right)=-w\left(\frac{\partial \pi}{\partial t}\right)+w(d s)$. From this it follows that 


$$
\begin{gathered}
w_{j}(L)=\left(-w\left(\frac{\partial \pi}{\partial t}\right)+w(d s)\right) e_{j}+e_{j}-1+d_{j} \text {. Next, } \\
w_{j}(R)=-\sum_{i \neq j} w_{j}\left(\pi_{i}\right)+w_{j}\left(\left|\begin{array}{ll}
\frac{\partial s}{\partial u} & \frac{\partial s}{\partial v} \\
\frac{\partial t}{\partial u} & \frac{\partial t}{\partial v}
\end{array}\right|\right)-w_{j}\left(\frac{\partial \pi_{j}}{\partial v}\right)+w_{j}(d u) .
\end{gathered}
$$

Thus, we have

$$
\left(-w\left(\frac{\partial \pi}{\partial t}\right)+w(d s)\right) e_{j}+e_{j}-1+d_{j}=-\sum_{i \neq j} w_{j}\left(\pi_{i}\right)+w_{j}\left(\begin{array}{ll}
\frac{\partial s}{\partial u} & \frac{\partial s}{\partial v} \\
\frac{\partial t}{\partial u} & \frac{\partial t}{\partial v}
\end{array} \mid\right)-w_{j}\left(\frac{\partial \pi_{j}}{\partial v}\right)+w_{j}(d u) .
$$

We apply the analytic adjunction formula to both sides, and apply Corollary 1.1.1] on the right-hand side:

$$
(-2 \delta(\mathcal{O} / \pi)+1) e_{j}+d_{j}=1-\sum_{i \neq j}\left(\pi_{i} . \pi_{j}\right)+\left(\beta^{\prime} . \pi_{j}\right)-2 \delta\left(\mathcal{O}^{\prime} / \pi_{j}\right),
$$

where $\beta^{\prime}$ is an equation of the ramification divisor in $\mathcal{O}^{\prime} / \mathcal{O}$. Finally, we sum over $j$ :

$$
-n\left(\operatorname{sing}_{\mathcal{O}}^{t} \pi-1\right)+\sum_{j} d_{j}=-\left(\operatorname{sing}_{\mathcal{O}^{\prime}}^{t} \pi-1\right)+(\beta . \pi) .
$$

Definition of $\lambda_{f}$. For the branch divisor of $\mathcal{O}^{\prime} / \mathcal{O}$ (viewed as a closed subscheme in Spec $\mathcal{O}$ ), denote by $\beta_{i}$ the equations of its prime components and by $b_{i}$ their multiplicities. We take an element $f \in \mathcal{O}$ that is a product of pairwise nonassociated prime elements in $\mathcal{O}$ such that $\operatorname{sing}_{\mathcal{O}}^{w} f<\infty$ and none of the $\beta_{i}$ divides $f$. Let $f=\pi_{1}^{\prime} \ldots \pi_{r}^{\prime}$ be a factorization of $f$ in $\mathcal{O}^{\prime}$. Every $\pi_{i}^{\prime}$ divides exactly one prime divisor $\pi_{i}$ of $f$ in $\mathcal{O}$; we denote by $A_{i}^{\prime}$ and $A_{i}$ the integral closures of $\mathcal{O}^{\prime} / \pi_{i}^{\prime}$ and $\mathcal{O} / \pi_{i}$, respectively. We define

$$
\lambda_{f}\left(\mathcal{O}^{\prime} / \mathcal{O}\right)=\sum_{i} b_{i}\left(1-d\left(f, \beta_{i}\right)\right)-(n-1)-\sum_{i=1}^{r} d\left(A_{i}^{\prime} \mid A_{i}\right)+\operatorname{sing}_{\mathcal{O}^{\prime}}^{w} f-n \operatorname{sing}_{\mathcal{O}}^{w} f
$$

Conjecture 4.2. Assume that there is no ferocious ramification in $\mathcal{O}^{\prime} / \mathcal{O}$. Let $f, f^{\prime} \in$ $\mathcal{O}$ be elements such that $\lambda_{f}\left(\mathcal{O}^{\prime} / \mathcal{O}\right)$ and $\lambda_{f^{\prime}}\left(\mathcal{O}^{\prime} / \mathcal{O}\right)$ are defined. Then $\lambda_{f}\left(\mathcal{O}^{\prime} / \mathcal{O}\right)=$ $\lambda_{f^{\prime}}\left(\mathcal{O}^{\prime} / \mathcal{O}\right)$.

Example. An ample series of examples can be constructed if for the role of $\mathcal{O}^{\prime}$ we take the integral closure of $\mathcal{O}$ in the extension of the fraction field of $\mathcal{O}$ given by the equation $x^{p}-x=t^{-p m+1} u^{-p n}$, where $t, u$ are fixed regular local parameters in $\mathcal{O}$, and $m, n$ are nonnegative integers. In is easily seen that such an $\mathcal{O}^{\prime}$ is always regular.

In this paper we only consider an example of a nonexceptional point on a component of the branch divisor without ferocious ramification. For this, take $m>0$ and $n=0$. (The notion of an exceptional point is due to Deligne [D]; see also Brylinski $[\mathrm{Br}$. Roughly speaking, these are the points on the ramification divisor where the codimension 2 ramification invariants take their nongeneric values.)

Let $y=t^{m} x$. Then $y^{p}-t^{(p-1) m} y=t$, whence $\mathcal{O}^{\prime} \supset \mathcal{O}[y]=k[[y, u]]$. Since $\mathcal{O}[y]$ is regular, we have $\mathcal{O}[y]=\mathcal{O}^{\prime}$, and

$$
t=y^{p}-y^{p+(p-1)(p m-1)}+O\left(y^{p+2(p-1)(p m-1)}\right) .
$$

The branch divisor $B$ consists of one component $t=0$, and $b_{1}=(p-1) p m$.

Let $f=t-u^{i}, i>0,(i, p)=1$. Then the expansion of $f$ in $k[[y, u]]$ is

$$
f=F(y, u)=y^{p}-y^{p+(p-1)(p m-1)}-u^{i}+O\left(y^{p+2(p-1)(p m-1)}\right) .
$$


We have

$$
\begin{aligned}
\operatorname{sing}_{\mathcal{O}^{\prime}}^{w} f & =d(f, y)-d(y, f)-d\left(f, \frac{\partial f}{\partial u}\right)+d\left(y, \frac{\partial f}{\partial u}\right) \\
& =0-0-(i-1) d(k[[y]] \mid k[[F(y, 0)]])+0 \\
& =(i-1)(p-1)(p m-1) .
\end{aligned}
$$

It follows that

$$
\begin{aligned}
\lambda_{f}\left(\mathcal{O}^{\prime} / \mathcal{O}\right) & =(p-1) p m-(p-1)-d(k[\widetilde{[y, u]]} / f \mid k[[u]])+(i-1)(p-1)(p m-1) \\
& =(p-1)(p m-1)-i(p-1)(p m-1)+(i-1)(p-1)(p m-1)=0 .
\end{aligned}
$$

\section{§5. SEVERI'S FORMUla}

In this section, $S$ is a regular geometrically irreducible complete surface over a perfect field $k ; K$ is the field of functions on $S$.

Lemma 5.1. Let $s, t$ be regular local parameters at $P \in S$. Then for any point $P^{\prime}$ in some neighborhood of $P$, the functions $s-s\left(P^{\prime}\right)$ and $t-t\left(P^{\prime}\right)$ are regular local parameters at $P^{\prime}$.

Proof. Let $U$ be a neighborhood of $P$ such that $s$ and $t$ are regular functions on $U$. Consider the morphism $f: U \rightarrow \mathbb{A}_{k}^{2}$ determined by the pair of functions $s, t$. Obviously, $f$ is unramified at $P$, whence $f$ is also unramified at all points of some neighborhood of $P$.

Let $\omega$ be a nonzero rational 1-differential on $S$, i.e., $\omega \in \Omega_{K / k}$, and $\omega \neq 0$. We define a divisor $(\omega)$ and a 0 -cycle $\langle\omega\rangle$ on $S$.

Let $P \in S$, and let $s, t$ be regular local parameters at $P$. Then $\omega=f_{P} \cdot\left(a_{P} d s+b_{P} d t\right)$, where $a_{P}$ and $b_{P}$ are coprime in $\mathcal{O}_{S, P}$, and $f_{P} \in K$. Then $(\omega)$ is defined as the divisor on $S$ such that in a neighborhood of any closed point $P$ it coincides with the divisor of the function $f_{P}$; this can be done in view of Lemma 5.1. Finally, by definition,

$$
\langle\omega\rangle=\sum_{P \in S_{0}} \operatorname{dim}_{k} \mathcal{O}_{S, P} /\left(a_{P}, b_{P}\right) \cdot P .
$$

Theorem 5.2. In the group $A_{0}(S)$ we have

$$
c_{2, S}=\langle\omega\rangle+(\omega) \cdot K_{S}-(\omega) \cdot(\omega) .
$$

Proof. See $\left[\mathrm{Y}\right.$, Corollary to Theorem $\left.2^{\prime}\right]$, or $[\mathrm{K}]$. See also the proof involving higher adèles in $[\mathrm{P}$.

\section{§6. Computation of the second Chern Class with the help OF A PENCIL OF CURVES}

Let $S$ be as in the preceding section; $k$ is assumed to be algebraically closed. Here a pencil of curves on $S$ is treated as a dominant rational map of $S$ in $\mathbb{P}_{k}^{1}$. In other words, this is a surjective morphism

$$
\mathcal{C}: S \backslash B \rightarrow \mathbb{P}_{k}^{1}
$$

that cannot be extended to any point of $B ; B$ is a closed subset of $S$, the so-called set of base points of a given pencil of curves. Theorem 3 in [Sh, Chapter II, $\S 3$ ] implies that $\operatorname{dim} B=0$.

We shall consider only pencils $\mathcal{C}$ of curves satisfying the following condition:

(*) the fiber of $\mathcal{C}$ over any point $s \in \mathbb{P}_{k}^{1}$ is a reduced subscheme. 
The closure of $\mathcal{C}^{-1}(s) \subset S \backslash B$ in $S$ will be denoted by $\mathcal{C}_{s}$. The theorem on dimension of fibers of a morphism (see, e.g., [H, Chapter II, Example 3.22]) implies that $\mathcal{C}_{s}$ is of pure dimension 1 . The schemes $\mathcal{C}_{s}$ are called curves in $\mathcal{C}$.

It is easily seen that all curves in $\mathcal{C}$ belong to one and the same divisor class on $S$. Therefore, for an arbitrary divisor $D$ on $S$ the intersection multiplicity $\left(\mathcal{C}_{s} \cdot D\right)$ is independent of $s$; it will be denoted simply by (C.D).

We require also that the following condition be fulfilled:

(**) for any base point $b$ all curves in $\mathcal{C}$ pass through $b$ and any two of them meet at $b$ transversally.

If $s=\mathcal{C}(P)$, the curve $\mathcal{C}_{s}$ will be denoted also by $\mathcal{C}_{P}$.

For $P \in S \backslash B$, we put $\operatorname{sing}_{P}^{w} \mathcal{C}=\operatorname{sing}_{\mathcal{O}_{S, P}}^{w}\left(t_{s} \circ \mathcal{C}\right)$ and $\operatorname{sing}_{P}^{t} \mathcal{C}=\operatorname{sing}_{\widehat{\mathcal{O}_{S, P}}}^{t}\left(t_{s} \circ \mathcal{C}\right)$, where $s=\mathcal{C}(P)$ and $t_{s}$ is a local parameter at $s$. Clearly, $\operatorname{sing}_{P}^{t} \mathcal{C}$ is independent of $t_{s}$. Remark 3.0 .1 shows that $\operatorname{sing}_{P}^{w} \mathcal{C}$ is also independent of $t_{s}$. Our third requirement is as follows.

$(* * *) \operatorname{sing}_{P}^{w} \mathcal{C}<\infty$ for all $P \in S \backslash B$.

We say that a pencil $\mathcal{C}$ on $S$ is separable if it satisfies conditions $(*),(* *)$, and (***).

Proposition 6.1. Let $S$ be a regular projective surface, and let $C_{1}, \ldots, C_{n}$ be prime divisors. Then there exists a pencil of curves $\mathcal{C}$ on $S$ with the set of base points $B$ such that:

- for any $i$ we have $B \cap C_{i}=\varnothing$;

- for any $i, \mathcal{C}$ induces a separable morphism $C_{i} \rightarrow \mathbb{P}_{k}^{1}$;

$-\mathcal{C}$ is separable.

Proof. The proposition is obvious in the case of $S=\mathbb{P}_{k}^{2}$; the general case can easily be derived by applying the following two lemmas.

Lemma 6.2. Under the conditions of Proposition 6.1. $S$ admits a finite separable morphism $g$ onto $\mathbb{P}_{k}^{2}$ such that $C_{1}, \ldots, C_{n}$ are not components of its ramification divisor $R_{g}$.

Proof. Assume that $S \subset \mathbb{P}^{N}$. We choose arbitrary closed points $P_{i} \in C_{i}, i=1, \ldots, n$, and let $H_{i}$ be the projective closure of the tangent plane to $S$ at $P_{i}$ embedded into $\mathbb{P}^{N}$. Choose a projective subspace $W \cong \mathbb{P}^{N-3}$ in $\mathbb{P}^{N}$ that meets neither $S$ nor any of $H_{i}$. Then the projection with the center $W$ determines a finite morphism of $S$ onto $\mathbb{P}^{2}$ unramified at any of the $P_{i}$.

Lemma 6.3. Let $h: T \rightarrow S$ be a finite separable morphism of surfaces, and let $\mathcal{C}$ be a separable pencil on $S$ such that the curves in $\mathcal{C}$ have no common components with the branch divisor $B_{h}$. Then the pencil $\mathcal{D}=\mathcal{C} \circ h$ on $T$ is also separable.

Proof. Let $b$ be any base point of $\mathcal{D}$. Since $h$ is unramified at $b$, regular local parameters at $h(b)$ generate the maximal ideal of $\mathcal{O}_{T, b}$. In other words, if two curves are regular at $h(b)$ and meet transversally at this point, the same is true for their preimages with respect to the point $b$. This proves that $\mathcal{D}$ satisfies $(* *)$. Condition $(*)$ can be verified by a similar argument applied to any regular point on a given curve in $\mathcal{D}$ such that $h$ is unramified at that point.

To verify condition $(* * *)$, it suffices to note that the Jacobi matrix of $h$ is of rank 2 at any point of $T$.

For a separable pencil $\mathcal{C}$ on $S$, we introduce the 0-cycle

$$
\operatorname{sing} \mathcal{C}=\sum_{P \in S \backslash B}\left(\operatorname{sing}_{P}^{t} \mathcal{C}+\operatorname{sing}_{P}^{w} \mathcal{C}\right) P .
$$


Since all curves in $\mathcal{C}$ belong to the same divisor class, their arithmetic genera are the same; this common value will be denoted by $p_{a}(\mathcal{C})$.

Proposition 6.4. Let $\mathcal{C}$ be a separable pencil of curves on $S$ with the set of base points $B$. Then

$$
\int c_{2, S}=\int \operatorname{sing} \mathcal{C}-\# B-4\left(p_{a}(\mathcal{C})-1\right) .
$$

Proof. We pick any rational function on the projective line such that its divisor of zeros as well as its divisor of poles has degree 1. (If we choose the point at infinity suitably, any such function is of the form $\lambda \frac{X-\alpha}{X-\beta}$ for some $\alpha, \beta, \lambda \in k$.) Let $f$ denote the inverse image of this function in $k(S)$. The idea of the proof is to apply Theorem 5.2 to $d f$.

The above explicit description of the original function implies that any other choice of such a function would change $f$ to $\frac{a f+b}{c f+d}$, where $a, b, c, d \in k, a d-b c \neq 0$. It follows that $\langle d f\rangle=\sum_{P \in S_{0}} n_{P} P$ is independent of this choice.

Now we compute $n_{P}$ for $P \notin B$. Let $D$ be any curve in $\mathcal{C}$ that is distinct from $\mathcal{C}_{P}$. If the divisor of $f$ is $\mathcal{C}_{P}-D$, then $f$ is a local equation of $\mathcal{C}_{P}$ at $P$. If $s$ and $t$ are local parameters at $P$, then $d f=\frac{\partial f}{\partial s} d s+\frac{\partial f}{\partial t} d t$. We have

$$
n_{P}=\left(\frac{\partial f}{\partial s} \cdot \frac{\partial f}{\partial t}\right)=\mu_{\widehat{\mathcal{O}_{S, P}}}(f)=\operatorname{sing}_{P}^{t} \mathcal{C}+\operatorname{sing}_{P}^{w} \mathcal{C}
$$

i.e., the 0 -cycles $\langle d f\rangle$ and $\operatorname{sing} \mathcal{C}$ have equal coefficients at $P$.

Now, let $P \in B$. For any choice of $f$, its divisor is $C-D$, where $C$ and $D$ are curves in $\mathcal{C}$ that meet transversally at $P$. Then $f$ can be written as $g / q$, where $g$ and $q$ are local equations of $C$ and $D$ at $P$. We have

$$
d f=\frac{g d q-q d g}{g^{2}}
$$

whence $n_{P}=1$.

Thus, we obtain

$$
\langle d f\rangle=\operatorname{sing} \mathcal{C}+\sum_{P \in B} P .
$$

Next, we compute $(d f)$. The divisor of $f$ is $C-D$, where $C$ and $D$ are some curves in $\mathcal{C}$; we shall prove that

$$
(d f)=-2 D .
$$

Take any $P \notin B$, and let $s$ and $t$ be regular local parameters. If $P \in C$, then, as we saw in the calculation of $\langle d f\rangle$, here $\frac{\partial f}{\partial s}$ and $\frac{\partial f}{\partial t}$ are coprime, i.e., $P \notin \operatorname{Supp}(d f)$. If $P \in D$, then $d f=-f^{2} \cdot d\left(f^{-1}\right)$ and, as above, $P \notin \operatorname{Supp}\left(d\left(f^{-1}\right)\right)$. Finally, if $P \notin C \cup D$, then $P$ belongs to the zero divisors of $f-f(P)$, and this case can be reduced to the case of $P \in C$ by replacing $f$ with $f-f(P)$.

Substituting (10) and (11) in the formula of Theorem [5.2, we obtain the following identity in $A_{0}(S)$ :

$$
c_{2, S}=\operatorname{sing} \mathcal{C}+\sum_{P \in B} B-2\left(C . K_{S}\right)-4(C . C),
$$

where $C$ is an arbitrary curve in $\mathcal{C}$. Clearly, condition $(* *)$ in the definition of a separable pencil implies that $(C . C)=\sum_{P \in B} B$. Therefore,

$$
c_{2, S}=\operatorname{sing} \mathcal{C}-\sum_{P \in B} B-2\left(C . K_{S}\right)-2(C . C) .
$$


Now it suffices to calculate the degrees and to apply the definition of the arithmetic genus.

We prove another property of pencils.

Proposition 6.5 (generalized Plücker equation). Let $\mathcal{C}$ be a pencil of curves on $S$ with the set of base points $B$, let $D$ be a reduced irreducible curve on $S$ that is not a component of any curve in $\mathcal{C}$, and let $D \cap B=\varnothing$. Assume that the restriction $\varphi$ of the morphism $\mathcal{C}$ to $D$ is a separable morphism of $D$ onto a projective line. Then

$$
\sum_{P \in D}\left(\left(\mathcal{C}_{P} . D\right)_{P}-\nu_{P}(D)+d_{P}\right)=2(\mathcal{C} . D)+2 p_{g}(D)-2,
$$

where $d_{P}$ is the sum of the wild differents of $\varphi$ at all points of the normalization of $D$ over $P$.

Proof. We denote by $\lambda: \tilde{D} \rightarrow D$ the normalization morphism. Let $P \in D$. For $P^{\prime} \in$ $\lambda^{-1}(D)$, let $e_{P^{\prime}}$ denote the ramification index of $\varphi \circ \lambda$ at $P^{\prime}$. Corollary 1.1.1 implies

$$
\sum_{P^{\prime} \in \lambda^{-1}(P)} e_{P^{\prime}}=\left(\mathcal{C}_{P} \cdot D\right)_{P}
$$

It is easily seen that $\operatorname{deg}(\varphi \circ \lambda)=(\mathcal{C} . D)$, and it remains to apply the Riemann-Hurwitz formula to the morphism $\varphi \circ \lambda$.

\section{§7. MORPHISMS OF SURFACES}

In this section we consider a finite separable morphism $h: T \rightarrow S$ of degree $n$.

It is known (see, e.g., [i] ) that in $A_{1}(T)$ we have

$$
K_{T}=h^{*} K_{S}+R_{f}
$$

A local equation of the ramification divisor can be determined as follows.

Lemma 7.1. Let $u, v$ be regular local parameters at $Q \in T$, and let $s, t$ be regular local parameters at $h(Q)$. Then $\left|\begin{array}{ll}\frac{\partial s}{\partial u} & \frac{\partial s}{\partial v} \\ \frac{\partial t}{\partial u} & \frac{\partial t}{\partial v}\end{array}\right|$ is a local equation of $R_{f}$ at $Q$.

Proof. In the exact sequence

$$
\Omega_{\mathcal{O}_{S, h(Q)} / k} \otimes_{\mathcal{O}_{S, h(Q)}} \mathcal{O}_{T, Q} \rightarrow \Omega_{\mathcal{O}_{T, Q} / k} \rightarrow \Omega_{\mathcal{O}_{T, Q} / \mathcal{O}_{S, h(Q)}} \rightarrow 0
$$

the first arrow is a homomorphism of two free $\mathcal{O}_{T, Q}$-modules with bases $d s, d t$ and $d u, d v$ (respectively); $d s$ and $d t$ are mapped to $\frac{\partial s}{\partial u} d u+\frac{\partial s}{\partial v} d v$ and $\frac{\partial t}{\partial u} d u+\frac{\partial t}{\partial v} d v$. Localizing with respect to all prime ideals of $\mathcal{O}_{T, Q}$ of height 1 , we see that for any prime divisor containing $Q$ with generic point $\eta$ we have

$$
l_{\mathcal{O}_{T, \eta}}\left(\Omega_{T / S, \eta}\right)=v_{\eta}\left(\left|\begin{array}{ll}
\frac{\partial s}{\partial u} & \frac{\partial s}{\partial v} \\
\frac{\partial t}{\partial u} & \frac{\partial t}{\partial v}
\end{array}\right|\right)
$$

where $v_{\eta}$ is the valuation of $k(T)$ associated with $\eta$.

We fix a pencil $\mathcal{C}$ as in Proposition 6.1 and denote by $\mathcal{D}$ the pencil of curves $\mathcal{C} \circ h$ on $T$ (the components of $B_{h}$ are taken for the role of $C_{1}, \ldots, C_{n}$ ), and by $B$ and $B^{\prime}$ the sets of base points of $\mathcal{C}$ and $\mathcal{D}$, respectively.

It is clear that $\# B^{\prime}=n \cdot \# B$. Therefore, by Proposition 6.4

$$
\chi_{T}-n \chi_{S}=\int \operatorname{sing} \mathcal{D}-n \int \operatorname{sing} \mathcal{C}-2\left(\left(2 p_{a}(\mathcal{D})-2\right)-n\left(2 p_{a}(\mathcal{C})-2\right)\right) .
$$


Lemma 7.2 (Zeuthen). Let $C$ be a divisor on $S$, and let $D=h^{*} C$. Then

$$
\left(2 p_{a}(D)-2\right)-n\left(2 p_{a}(C)-2\right)=\left(C . B_{h}\right) .
$$

Proof. Using (12) and the projection formula, we obtain

$$
\begin{aligned}
2 p_{a}(D)-2 & =(D \cdot D)+\left(K_{T} \cdot D\right) \\
& =n(C \cdot C)+\left(h^{*} K_{S} \cdot D\right)+\left(R_{h} \cdot D\right) \\
& =n(C \cdot C)+n\left(K_{S} \cdot C\right)+\left(B_{h} \cdot C\right) \\
& =n\left(2 p_{a}(C)-2\right)+\left(B_{h} \cdot C\right) .
\end{aligned}
$$

By definition, we have

$$
\begin{aligned}
& \int \operatorname{sing} \mathcal{D}-n \int \operatorname{sing} \mathcal{C} \\
& =\sum_{P \in S \backslash B}\left(\sum_{Q \in h^{-1}(P)} \operatorname{sing}_{Q}^{t} \mathcal{D}_{Q}-n \operatorname{sing}_{P}^{t} \mathcal{C}_{P}+\sum_{Q \in h^{-1}(P)} \operatorname{sing}_{Q}^{w} \mathcal{D}_{Q}-n \operatorname{sing}_{P}^{w} \mathcal{C}_{P}\right) .
\end{aligned}
$$

For an effective divisor $C$ on $S$ without multiple components and without common components with $B_{h}$, and for a point $Q$ on $T$, we introduce the notation

$$
d_{Q}(C)=\sum_{\pi, \pi^{\prime}} d\left(\left(\widehat{\mathcal{O}_{T, Q}} / \pi^{\prime}\right) \mid\left(\widehat{\mathcal{O}_{S, h(Q)}} / \pi\right)\right),
$$

where $\pi$ runs over the nonassociated prime divisors in $\widehat{\mathcal{O}_{S, h(Q)}}$ of a local equation of $C$ at $h(Q)$, and $\pi^{\prime}$ runs over the nonassociated prime divisors of $\pi$ in $\widehat{\mathcal{O}_{T, Q}}$.

Proposition 7.3. Let $C$ be an effective divisor on $S$ without multiple components and without common components with $B_{h}$, let $P$ be any point of $\operatorname{Supp} C$, and let $f$ be a local equation of $C_{h(Q)}$ at $h(Q)$. Then

$$
\sum_{Q \mapsto P} \operatorname{sing}_{\widehat{\mathcal{O}_{T, Q}}}^{t}(f \circ h)-n \operatorname{sing}_{\widehat{\mathcal{O}_{S, P}}}^{t} f=\left(C . B_{h}\right)_{P}-\left(n-\# h^{-1}(P)\right)-\sum_{Q \mapsto P} d_{Q}(C) .
$$

Proof. We can immediately reduce the proposition to a similar statement in which $S$ is the spectrum of a complete 2-dimensional regular local ring. Next, it reduces to the case where $T$ is connected, i.e., is also the spectrum of a complete 2-dimensional regular local ring.

Let $C=\sum C_{i}$, where the $C_{i}$ are prime divisors. For $i \neq j$ we have

$$
n\left(C_{i} \cdot C_{j}\right)_{P}=\sum_{Q \in h^{-1}(P)}\left(h^{*} C_{i} \cdot h^{*} C_{j}\right)_{Q} .
$$

Therefore, the proposition reduces immediately to the case where $C$ is a prime divisor, and this case is none other than Proposition 4.1.

Theorem 7.4. Let $B_{h}=\sum b_{i} B_{i}$ be the branch divisor of $h$. Let $\mathcal{C}$ be any separable pencil on $S$ such that none of the $B_{i}$ is a component of any curve in $\mathcal{C}$. Then

$$
\chi_{T}-n \chi_{S}=\sum_{i} b_{i}\left(2 p_{g}\left(B_{i}\right)-2\right)+\sum_{Q} \lambda_{f}\left(\widehat{\mathcal{O}_{T, Q}} / \widehat{\mathcal{O}_{S, h(Q)}}\right),
$$

where $f$ is a local equation of $\mathcal{C}_{h(Q)}$ at $h(Q)$. 
Proof. Proposition 7.3 implies

$$
\begin{aligned}
\int \operatorname{sing} \mathcal{D}-n \int \operatorname{sing} \mathcal{C}=\sum_{P \in S \backslash B}\left(\left(\mathcal{C}_{P} \cdot B_{h}\right)_{P}-\left(n-\# h^{-1}(P)\right)-\sum_{Q \mapsto P} d_{Q}\left(\mathcal{C}_{P}\right)\right. \\
\left.+\sum_{Q \mapsto P} \operatorname{sing}_{Q}^{w} \mathcal{D}_{Q}-n \operatorname{sing}_{P}^{w} \mathcal{C}_{P}\right) P .
\end{aligned}
$$

Together with (13) and Lemma 7.2, this yields

$$
\begin{aligned}
& \chi_{T}-n \chi_{S}=-2\left(\mathcal{C} \cdot B_{h}\right)+\sum_{P \in S \backslash B}\left(\left(\mathcal{C}_{P} \cdot B_{h}\right)_{P}-\left(n-\# h^{-1}(P)\right)-\sum_{Q \mapsto P} d_{Q}\left(\mathcal{C}_{P}\right)\right. \\
& \left.+\sum_{Q \mapsto P} \operatorname{sing}_{Q}^{w} \mathcal{D}_{Q}-n \operatorname{sing}_{P}^{w} \mathcal{C}_{P}\right)
\end{aligned}
$$

Therefore,

$$
\begin{aligned}
\chi_{T}-n \chi_{S}=-2 & \sum_{i} b_{i}\left(\mathcal{C} \cdot B_{i}\right)+\sum_{i} \sum_{P \in S} b_{i}\left(\left(\mathcal{C}_{P} \cdot B_{i}\right)_{P}-\nu_{P}\left(B_{i}\right)\right) \\
& +\sum_{P}\left(\sum_{i} b_{i} \nu_{P}\left(B_{i}\right)-\left(n-\# h^{-1}(P)\right)-\sum_{Q \mapsto P} d_{Q}\left(\mathcal{C}_{P}\right)\right. \\
& \left.+\sum_{Q \mapsto P} \operatorname{sing}_{Q}^{w} \mathcal{D}_{Q}-n \operatorname{sing}_{P}^{w} \mathcal{C}_{P}\right) .
\end{aligned}
$$

Note that for any $i$ the morphism $\varphi_{i}: B_{i} \rightarrow \mathbb{P}_{k}^{1}$ determined by $\mathcal{C}$ is separable. Indeed, if $P \in B_{i}$ is any point of $B_{i}$ regular on $B_{h}$ and such that $\mathcal{C}_{P}$ meets $B_{h}$ at $P$ transversally, then a local parameter at $\varphi_{i}(P)$ on $\mathbb{P}_{k}^{1}$ is mapped to a local parameter at $P$ on $B_{i}$. Thus, we can apply Proposition 6.5, obtaining

$$
\begin{aligned}
\chi_{T}-n \chi_{S}= & \sum_{i} b_{i}\left(2 p_{g}\left(B_{i}\right)-2\right) \\
+ & \sum_{P}\left(\sum_{i}\left(-b_{i} \sum_{P^{\prime}} d\left(\widehat{\mathcal{O}_{\widetilde{B_{i}}, P^{\prime}}} \mid \widehat{\mathcal{O}_{\mathbb{P}_{k}^{1}, \varphi_{i}}(P)}\right)+b_{i} \nu_{P}\left(B_{i}\right)\right)\right. \\
& \left.\quad\left(n-\# h^{-1}(P)\right)-\sum_{Q \mapsto P} d_{Q}\left(\mathcal{C}_{P}\right)+\sum_{Q \mapsto P} \operatorname{sing}_{Q}^{w} \mathcal{D}_{Q}-n \operatorname{sing}_{P}^{w} \mathcal{C}_{P}\right),
\end{aligned}
$$

where $P^{\prime}$ runs over the points over $P$ of the normalization $\widetilde{B_{i}}$ of $B_{i}$. Obviously,

$$
n-\# h^{-1}(P)=\sum_{Q \mapsto P}\left(n_{Q}-1\right),
$$

where $n_{Q}$ is the degree of $\widehat{\mathcal{O}_{T, Q}}$ over $\widehat{\mathcal{O}_{S, P}}$. It remains to rewrite $b_{i}$ as a sum over $Q \mapsto P$ of similar infinitesimal terms. This completes the proof of Theorem 7.4 .

\section{REFERENCES}

[Br] J.-L. Brylinski, Théorie du corps de classes de Kato et revêtements abéliens de surfaces, Ann. Inst. Fourier (Grenoble) 33 (1983), no. 3, 23-38. MR0723946 (85f:11088)

[D] P. Deligne, Letter to L. Illusie of 28.11.76, not published.

[F] W. Fulton, Intersection theory, 2nd ed., Ergeb. Math. Grenzgeb. (3) Ser. Modern Surveys in Math., Bd. 2, Springer-Verlag, Berlin, 1998. MR.1644323 (99d:14003)

[GK] G.-M. Greuel and H. Kröning, Simple singularities in positive characteristic, Math. Z. 203 (1990), no. 2, 339-354. MR1033443 (90k:14001) 
[H] R. Hartshorne, Algebraic geometry, Grad. Texts in Math., Bd. 52, Springer-Verlag, New YorkHeidelberg, 1977. MR0463157 (57:3116)

[L] G. Laumon, Semi-continuité du conducteur de Swan (d'après P. Deligne), Astérisque 83 (1981), 173-219. MR0629128 (83g:14007)

[K] H. Kurke, Vorlesungen über algebraische Flächen, Teubner-Texte Math., Bd. 43, BSB B. G. Teubner Verlagsgesellschaft, Leipzig, 1982. MR0680403 (84e:14029)

[Ii] S. Iitaka, Algebraic geometry. An introduction to birational geometry of algebraic varieties, Grad. Texts in Math., Bd. 76, Springer-Verlag, New York-Berlin, 1982. MR0637060 (84j:14001)

[Iv] B. Iversen, Numerical invariants and multiple planes, Amer. J. Math. 92 (1970), 968-996. MR0296074 (45:5135)

[P] A. N. Parshin, Chern classes, adèles and L-functions, J. Reine Angew. Math. 341 (1983), 174-192. MR0697316 (85c:14015)

[Sa] P. Samuel, Singularités des variétés algébriques, Bull. Soc. Math. France 79 (1951), 121-129. MR0052832(14:683a)

[Se] J. P. Serre, Corps locaux, Actualités Sci. Indust., No. 1296, Hermann, Paris, 1962. MR0150130 $(27: 133)$

[Sh] I. R. Shafarevich, Basic algebraic geometry. Vols. 1, 2, 2nd ed., "Nauka", Moscow, 1988; English transl., Springer-Verlag, Berlin, 1994. MR0969372 (90g:14001) MR0979764 (90g:14002) MR.1328833 (95m:14001) MR.1328834 (95m:14002)

[Y] H. Yamada, Rational sections and Chern classes of vector bundles, J. Math. Kyoto Univ. 6 (1967), no. 2, 295-312. MR.0209298 (35:196)

Department of Mathematics and Mechanics, St. Petersburg State University, Universitetski ̌ Prospekt 28, Stary ̌ Peterhof, St. Petersburg 198504, Russia

E-mail address: igor.zhukov@mail.ru

Received 23/MAY/2007

Translated by THE AUTHOR 